\title{
Erythrocyte Membrane Modified Janus Polymeric Motors for Thrombus Therapy
}

\author{
Jingxin Shao, Mona Abdelghani, Guizhi Shen, Shoupeng Cao, David S. Williams, ${ }^{\oplus}$ \\ and Jan C. M. van Hest*0
}

Bio-Organic Chemistry, Eindhoven University of Technology, P.O. Box 513 (STO 3.41), 5600 MB Eindhoven, The Netherlands

\author{
Supporting Information
}

\begin{abstract}
We report the construction of erythrocyte membrane-cloaked Janus polymeric motors (EM-JPMs) which are propelled by near-infrared (NIR) laser irradiation and are successfully applied in thrombus ablation. Chitosan (a natural polysaccharide with positive charge, $\mathrm{CHI}$ ) and heparin (glycosaminoglycan with negative charge, Hep) were selected as wall materials to construct biodegradable and biocompatible capsules through the layer-by-layer self-assembly technique. By partially coating the capsule with a gold $\mathrm{Au}$ ) layer through sputter coating, a NIRresponsive Janus structure was obtained. Due to the asymmetric distribution of $\mathrm{Au}$, a local thermal gradient was generated upon NIR irradiation, resulting in the movement of the JPMs through the self-thermophoresis effect. The reversible "on/ off" motion of the JPMs and their motile behavior were easily tuned by the incident NIR laser intensity. After biointerfacing the Janus capsules with an erythrocyte membrane, the EM-JPMs displayed red blood cell related properties, which enabled them to move efficiently in relevant biological environments (cell culture, serum, and blood). Furthermore, this therapeutic platform exhibited excellent performance in ablation of thrombus through photothermal therapy. As man-made micromotors, these biohybrid EM-JPMs hold great promise of navigating in vivo for active delivery while overcoming the drawbacks of existing synthetic therapeutic platforms. We expect that this biohybrid motor has considerable potential to be widely used in the biomedical field.
\end{abstract}

KEYWORDS: Janus polymeric motors, erythrocyte membrane, biostealth coating, biocompatibility, anti-thrombus

$\mathrm{M}$ an-made micro/nanomotors with autonomous movement have recently drawn much attention. ${ }^{1-3}$ Over the past decade, impressive progress in designing powerful micro/nanomachines based on various propulsion mechanisms has led to advances in biomedical applications, such as drug delivery, ${ }^{4-6}$ precision surgery, ${ }^{7}$ cell sensing, ${ }^{8,9}$ diagnostics, and therapy. ${ }^{10,11}$ Although current powerful motors have been well designed, proper attention needs to be paid to overcome a number of main challenges for performing complex tasks in a physiologically relevant environment. Most motors are propelled by kinetic energy derived from chemical reactions, which often requires the usage of less compatible substrates such as hydrogen peroxide and hydrazine, with limited control over the directionality of movement. ${ }^{12}$ Light, magnetic field, ultrasound, and electrically driven motors are well-designed recently to overcome this obstacle. ${ }^{13-15}$ Furthermore, the majority of nano- and micromotors still rely on synthetic materials, although micro/ nanomotors themselves can be constructed out of naturally occurring building blocks. As a result, undesired immunogenicity and biofouling still present a tough challenge for these artificial motors, which hampers their performance in a clinical setting. Consequently, from a clinical point of view, it is necessary to explore the next generation of fuel-free self- propelled motors that have a clear biosafety profile as well as precisely controlled motion. Natural polysaccharides are an ideal candidate material for the construction of such drug delivery systems with improved behavior in a physiological environment. These polymers are nontoxic, biocompatible, and biodegradable. ${ }^{16}$ In addition, some of them, such as heparin, have therapeutic activity and can be used not only as building blocks but also as treatment modality. As the active component is an integral part of the particle structure, side effects induced by drug leaking will be effectively avoided.

In order to minimize undesired interactions with the immune system even further, particles can be decorated with a cellderived coating. As the most abundant cells in blood, erythrocytes with their physical and chemical properties are intrinsically biocompatible, biodegradable, and nonimmunogenic materials. ${ }^{17}$ Due to the protein makeup on their surface, erythrocytes display a long-circulation behavior within a physiological environment. ${ }^{18}$ However, conventional chemical or biological approaches would be impractical for mimicking

\footnotetext{
Received: March 8, 2018

Accepted: May 7, 2018

Published: May 7, 2018
} 

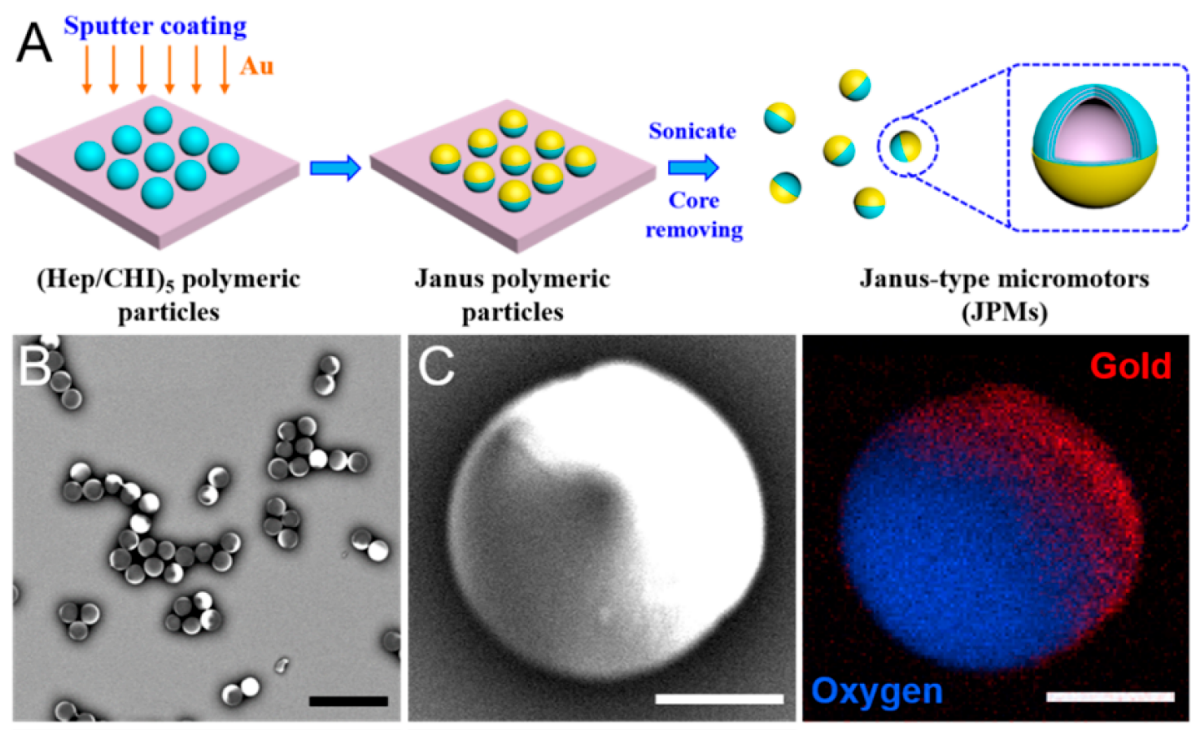

Janus polymeric

removing particles
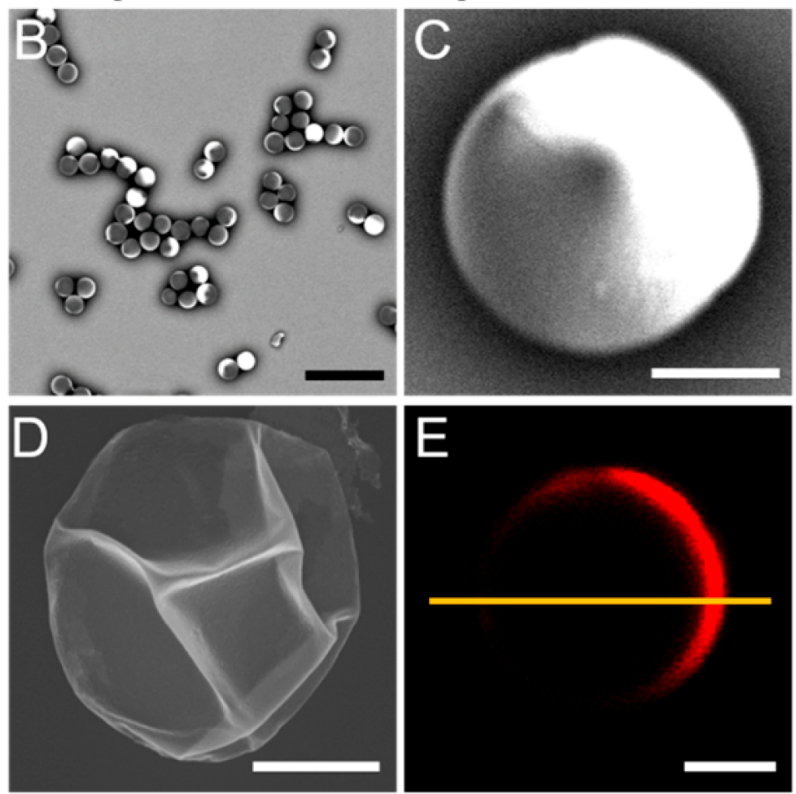

Janus-type micromotors (JPMs)
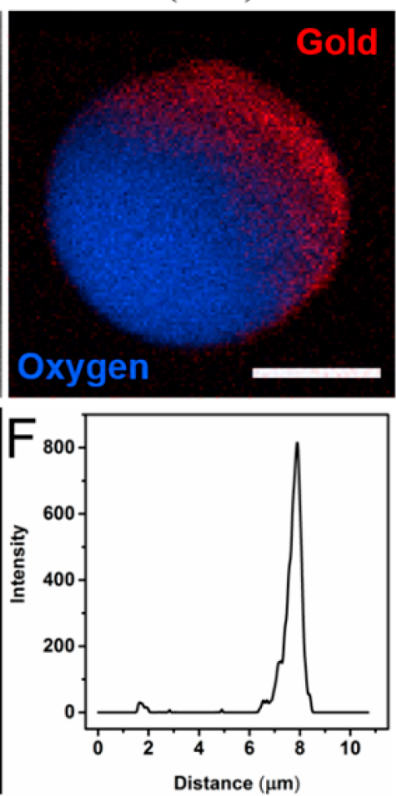

Figure 1. Preparation and characterization of biodegradable JPMs. (A) Schematic illustration of synthetic procedure of JPMs by combining the LbL self-assembly technique with the metal sputter-coating method. (B) Scanningn electron microscopy (SEM) image of as-prepared JPMs before dissolving the template. Scale bar $=20 \mu \mathrm{m}$. (C) Energy-dispersive X-ray spectroscopy elemental mapping analysis for gold and oxygen before removal of the template. Scale bar $=2 \mu \mathrm{m}$. (D) SEM image of JPMs after removing the template. Scale bar $=2 \mu \mathrm{m}$. (E) Confocal laser scanning microscopy image of JPMs. Rhodamine B was used as the fluorescence tag to prove that the Janus structure was obtained after coating with gold. Scale bar $=2 \mu \mathrm{m}$. (F) Corresponding fluorescence intensity profile of JPMs in panel E.

the complex membrane structure and physiological properties. A recent, highly promising approach to confer biomimetic surface properties onto nanoparticles is to decorate them with the isolated membrane of erythrocytes, or red blood cells (RBCs). ${ }^{19-22}$ By introducing a cell membrane coating, synthetic materials have shown capability for accomplishing comprehensive biological applications ranging from drug delivery, toxin absorption, and immune evasion. ${ }^{23}$ Hence, for synthetic motors, direct cloaking with a cell-derived membrane is a convenient way to install the desired behavior in a biological environment onto the particles. ${ }^{24-27}$ Considerable progress has been made on designing biomimetic inorganic particle-based motors, like RBC membrane-coated gold nanowire motors, $\mathrm{Mg}$ Janus motors, and platelet-membrane-cloaked magnetic helical nanomotors, ${ }^{28-30}$ whereas there are still no reports about combining this cell membrane coating strategy with polymer-based micro/nanomotors.

In this report, we describe the successful preparation of Janus-type polymeric micromotors (JPMs) composed of heparin (Hep) and chitosan ( $\mathrm{CHI}$ ), which are decorated with a stealth layer originating from an erythrocyte membrane. Due to the opposite surface charges, the two natural polymers can be assembled into capsules via a layer-by-layer (LbL) procedure. Here, Hep not only is a structural component of the capsule but also has biological function, in the sense that it can be used to prevent or dissolve blood clotting, or thrombosis. To ensure fuel-less propulsion, the LbL capsules are partially covered with a gold shell in order to employ nearinfrared (NIR) irradiation to achieve motion via the photothermal effect. Again, NIR irradiation has a double function, as it will not only be used for motion but can also be used for photothermal ablation of thrombus. The sensitivity to NIR irradiation is therefore explored to induce motor function in a biological environment such as blood and in a thrombus disease model to evaluate the efficacy of photothermal therapy. The in vitro experiments demonstrate that the combined properties of these JPMs provide a safe and efficient platform for thrombolytic therapy.

\section{RESULTS AND DISCUSSION}

Janus-type polyelectrolyte microcapsules composed of polysaccharides were prepared in three steps (Figure 1A). First, the LbL self-assembly technique allowed the construction of polymeric particles. Briefly, template silica particles (diameter $5 \mu \mathrm{m}$ ) were alternately exposed to $\mathrm{CHI}$ and Hep solutions under continuous shaking, leading to LbL assembly. After assembly of five double layers, the corresponding polymeric particles were washed three times to remove the unassembled polymers. During this process, surface charge reversal of the polymeric particles was monitored by dynamic light scattering (DLS). As shown in Figure S1, Hep and CHI were successfully assembled on the surface of the templates. 


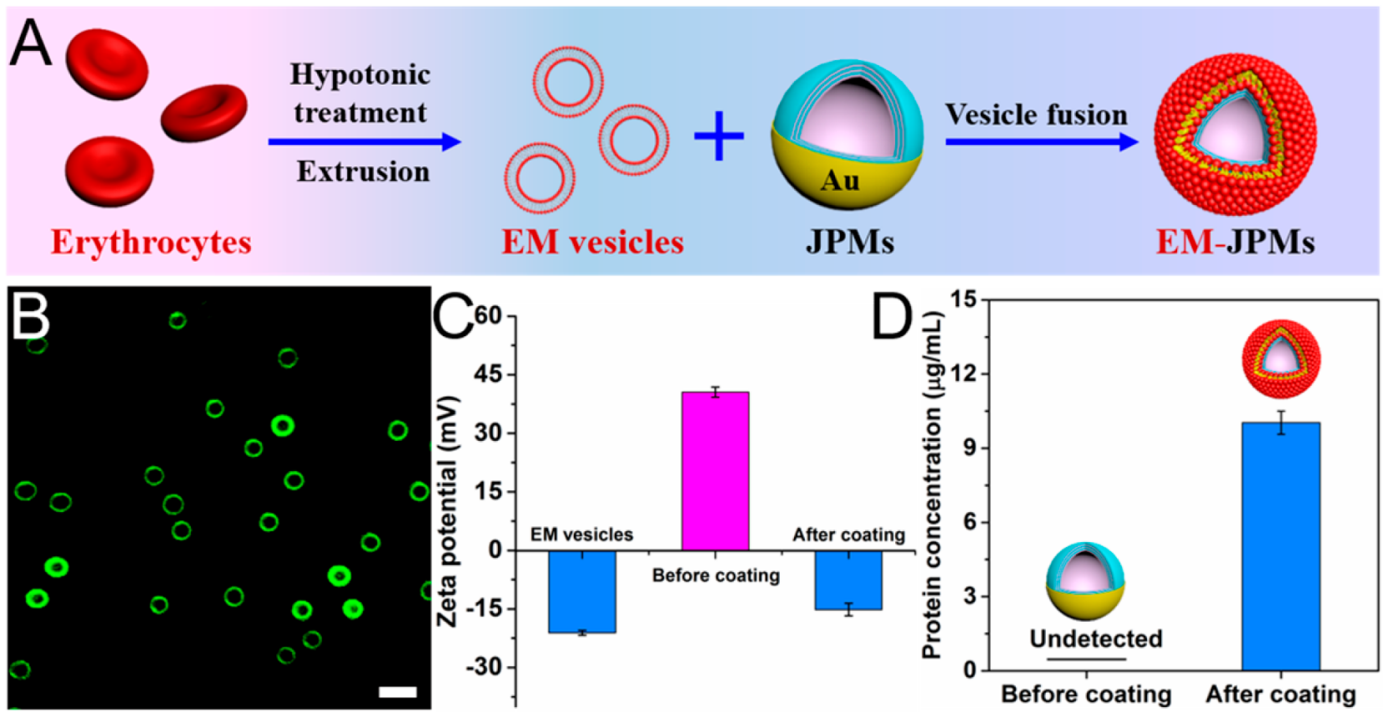

Figure 2. Modification of JPMs with an erythrocyte membrane (EM) by the vesicle fusion method. (A) Schematic illustration of the modification of gold-coated JPMs with an erythrocyte membrane coating. (B) CLSM images of JPMs after coating with the membrane. Green fluorescence corresponds to the Alexa Fluor 488 which stained the EM. Scale bar $=10 \mu \mathrm{m}$. (D) $\zeta$-Potential (mV) of EM vesicles and JPMs before and after coating with the cell membrane. (D) BCA assay for quantification of protein concentration of JPMs before and after coating.
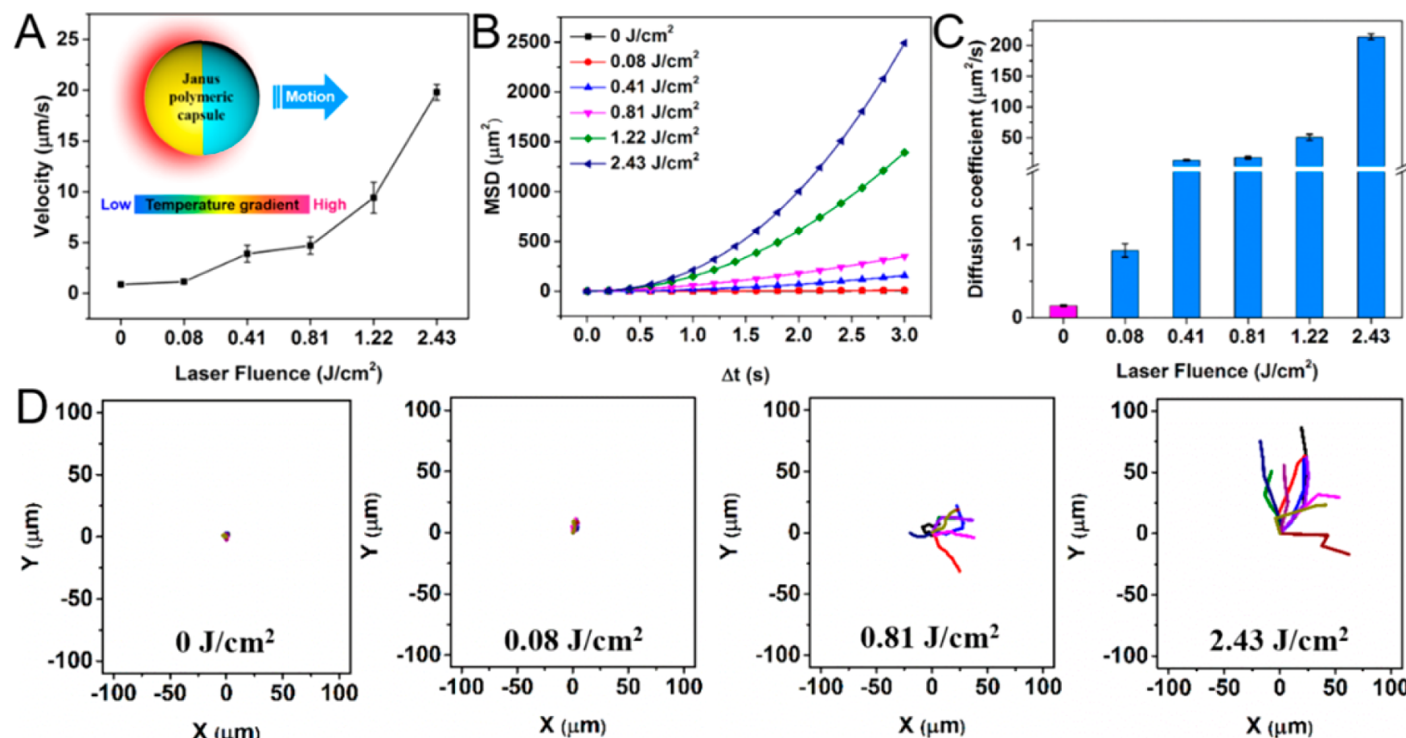

$\Delta t(s)$
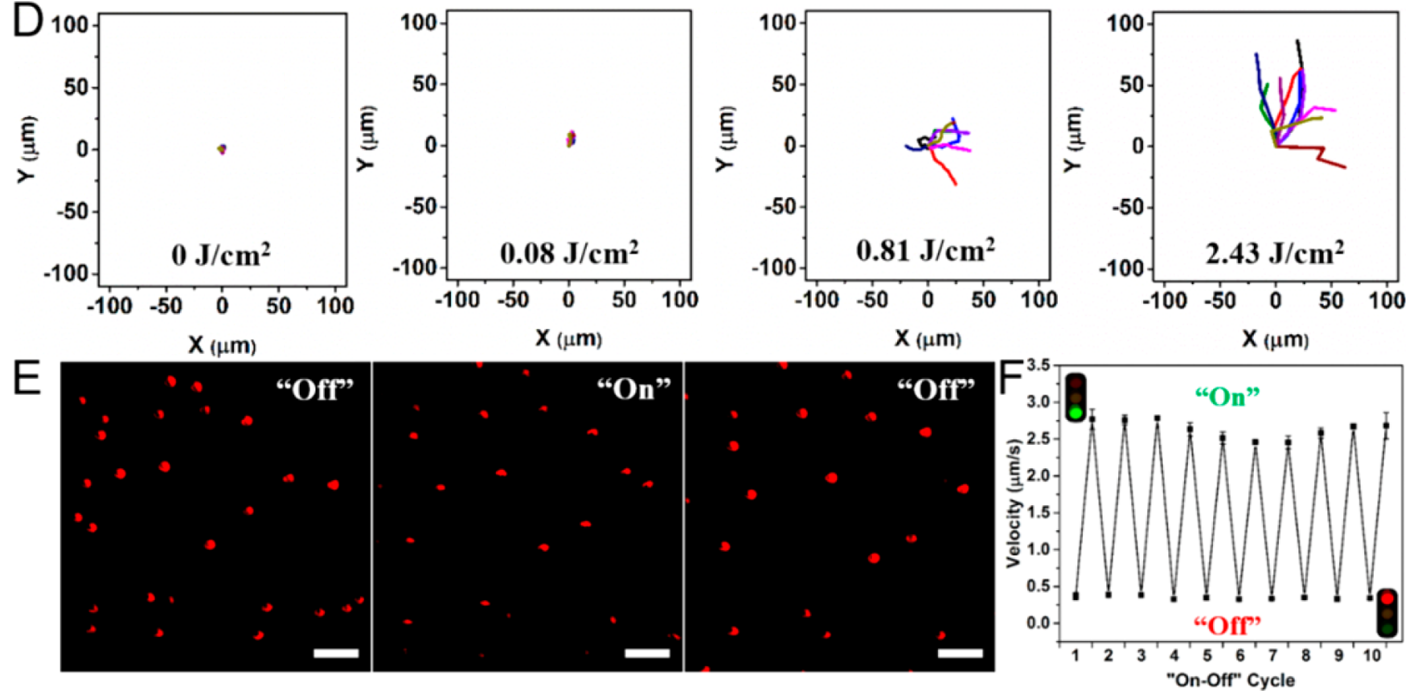

Figure 3. Movement analysis of EM-JPMs. (A) Velocity of EM-JPMs as a function of laser power. Inset image is the schematic representation of the movement of EM-JPMs driven by the NIR laser. (B) Mean square displacement (MSD) as a function of laser power. (C) Diffusion coefficient as a function of laser intensity. (D) Tracking trajectories of EM-JPMs as a function of laser fluence $\left(0,0.08,0.81\right.$, and $\left.2.43 \mathrm{~J} / \mathrm{cm}^{2}\right)$. (E) CLSM images of one cycle movement of EM-JPMs by illumination with $0.41 \mathrm{~J} / \mathrm{cm}^{2}$ laser. Scale bar $=20 \mu \mathrm{m}$. (F) Real time "on-off" velocity control by irradiation with or without NIR laser $(10$ cycles).

Second, in order to form the Janus structure, polymeric particles were deposited as a monolayer on the surface of a silicon wafer (Figure S2), and then a gold shell was introduced selectively on part of the particles via a sputter-coating approach (Figure 1B). Energy-dispersive X-ray spectroscopy (EDX) elemental mapping analysis for gold and oxygen (Figure 1C) confirmed the Janus morphology. Third, to form JPMs with a hollow structure, the templates were then removed, as 

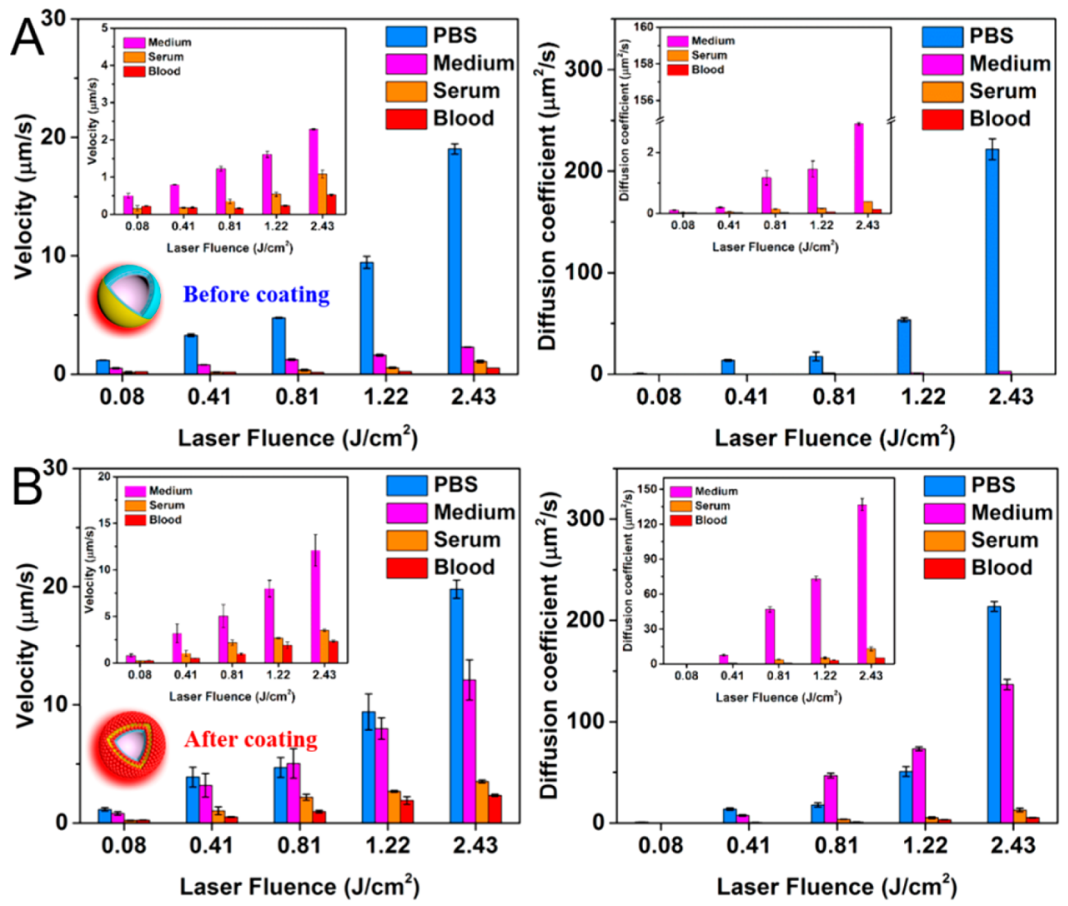

Figure 4. Differences in movement behavior of JPMs before and after coating with an erythrocyte membrane, as a function of solution (PBS, cell culture medium, serum, and blood). (A) Velocities and diffusion coefficients of JPMs before erythrocyte membrane camouflaging. (B) Velocities and diffusion coefficients of EM-JPMs.

shown in Figure 1D. The resulting Au-functionalized Janus structure was characterized by confocal laser scanning microscopy (CLSM), as presented in Figure 1E. Here, polymeric capsules were labeled by rhodamine B. After we coated half of the capsule with a shell of gold, half of the red fluorescence of the capsule had disappeared (Figure 1F).

Erythrocytes, as natural long-circulating delivery vehicles, are intrinsically biocompatible, biodegradable, and nonimmunogenic. ${ }^{31}$ Due to their surface makeup (a myriad of proteins, glycans, and acidic sialyl moieties), erythrocytes achieve longterm circulation in a physiological environment. ${ }^{32}$ By cloaking the JPMs with the isolated erythrocyte membrane, we expected that we could also introduce similar physicochemical characteristics and biological functions of erythrocytes that would otherwise be difficult to mimic. First, erythrocyte membrane vesicles were derived from natural erythrocytes (Figure S3). The as-prepared vesicles were then coated on the surface of JPMs by a vesicle fusion method, ${ }^{33,34}$ as shown in Figure $2 \mathrm{~A}$. Because the entire membrane was translocated, all biologically relevant surface moieties and membrane-bound proteins were also transferred to the surface of the JPMs. The successful transfer was demonstrated via a cell membrane targeting fluorescent dye (Wheat germ agglutinin, Alexa Fluor 488 conjugate), which could also stain the erythrocyte membranecoated JPMs (EM-JPMs, Figure 2B). Furthermore, $\zeta$-potential measurements by DLS also proved the successful coating with the cell membrane (Figure 2C), as the surface charge of the JPMs was changed from positive to negative and to a value comparable to one measured for the cell membrane vesicles. Next, a protein bicinchoninic acid (BCA) assay was used to quantify the protein content before and after membrane coating. As presented in Figure 2D, bare JPMs showed the absence of detectable protein. A significant increase in protein content was observed after the membrane fusion treatment. Meanwhile, SDS-PAGE was conducted to separate the associated cell membrane proteins of the EM-JPMs. Bare capsules and membrane vesicles as control groups were also analyzed (Figure S3C). EM-JPMs showed the typical bands that are similar to the membrane vesicles, confirming the presence of the corresponding membrane.

NIR laser-powered motors have been recognized for their great potential in biomedical applications. Recently, a carbonaceous nanobottle motor based on NIR-driven jet propulsion was designed, which relied on the photothermal effect of the carbon shell. ${ }^{35}$ Gold hybrid polymers have a similar photothermal effect, which makes them also suitable to be used as man-made motors. It has been demonstrated that laser-induced localized heating could be obtained by integrating plasmonic gold materials into LbL-assembled polymers. ${ }^{36-39}$ In the case of our JPMs, irradiation with a NIR laser led to the formation of a temperature gradient around the particles due to the asymmetric $\mathrm{Au}$ coating. As a result, the JPMs experienced a force moving toward the nonheated side. Consequently, they were able to display directed motion via the thermophoretic effect. ${ }^{40}$ The UV-vis-NIR spectra of the EM-JPMs in Figure S4A revealed that the maximum absorption peak of this structure was located in the NIR window, which is the key condition for obtaining the optimal photothermal conversion effect. ${ }^{41}$ Thus, two-photon confocal laser scanning microscopy (TP-CLSM) employing a NIR laser source with wavelength of $760 \mathrm{~nm}$ was used to induce and record the movement. The extent of movement was facilely tuned by the incident laser intensity, as shown in Figure 3A. The velocity of the EM-JPMs was linear to the NIR laser fluence. Additionally, the mean square displacement (MSD) versus $\Delta t$ and the diffusion coefficient $(D)(D=\mathrm{MSD} /(i \times \Delta t)$, where $i$ is the dimensional index and $\Delta t$ is the time interval) were calculated from the recorded video (Figure 3B,C). ${ }^{42-44}$ Furthermore, the tracking trajectory of the JPMs in phosphate-buffered saline (PBS) solution as a function of laser fluence was captured by ImageJ 

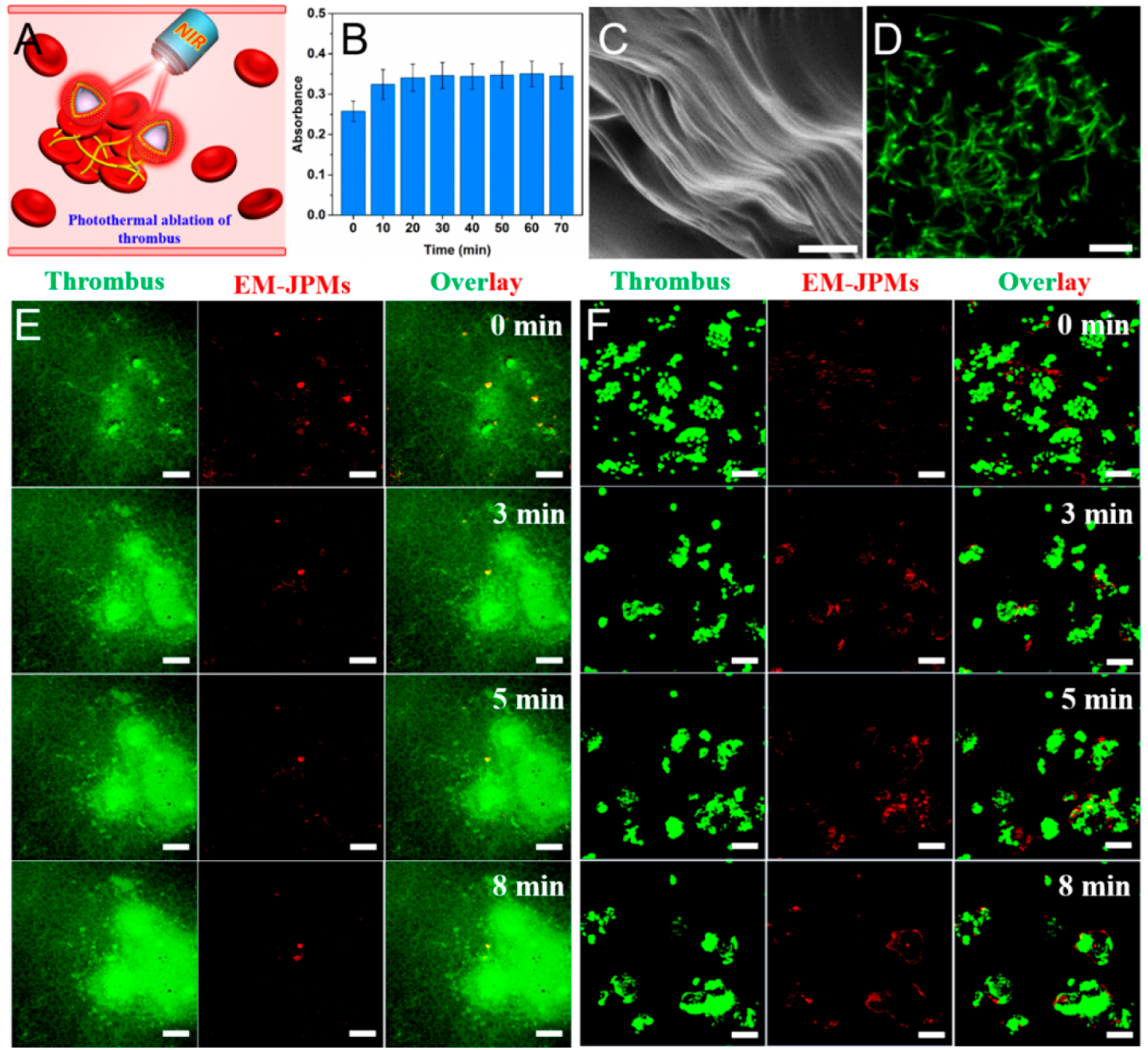

Figure 5. Characterization of photothermal ablation of thrombus. (A) Schematic illustration. (B) Turbidity assay using the UV absorbance at $340 \mathrm{~nm}$ during the formation of the thrombus model out of purified fibrinogen. (C) SEM image of the as-prepared thrombus model. Scale bar $=500 \mathrm{~nm}$. (D) CLSM image of the fluorescent thrombus prepared by Alexa Fluor 488-conjugated fibrinogen. Scale bar $=5 \mu \mathrm{m}$. (E) Timelapsed TP-CLSM images of fluorescent thrombus in the presence of EM-JPMs irradiated by a $760 \mathrm{~nm}$ NIR laser. Scale bar $=20 \mu \mathrm{m}$. (F) Timelapsed TP-CLSM images of fluorescent floating fibrin clots in the presence of EM-JPMs upon NIR irradiation $(760 \mathrm{~nm})$. Scale bar $=20 \mu \mathrm{m}$.

software and is plotted in Figure $3 \mathrm{D}$ and Figure S4B. Obviously, EM-JPMs display typical Brownian motion without illumination with the NIR laser (Video S1). In sharp contrast, with increasing NIR laser fluence, the EM-JPM trajectories were clearly extended (Video S2). The above-mentioned data, including velocity, MSD calculations, diffusion coefficients, and trajectories, confirmed that the movement of EM-JPMs can be easily tuned by the incident NIR laser power. In addition, 10 "off/on" cyclic tests of the movement were conducted to prove that this movement is repeatable and recyclable. As shown in Figure 3E,F, taken from Video S3, once the NIR laser was switched off, the EM-JPMs completely stopped their motion, whereas the motion was restarted immediately after the NIR laser source was switched on.

One of the main limitations of application of man-made motors in a biological environment is the reversible/irreversible adsorption of proteins and other biomolecules on the surface of motors, which can strongly affect the motion capability. The coating of the JPMs with the erythrocyte membrane should introduce antibiofouling properties and leave motion unaffected. $^{28,29,45}$ Hence, the propulsion performance and antibiofouling of EM-JPMs in several biological fluids (PBS, cell culture medium, serum, and blood) were tested. In PBS, coated and bare JPMs displayed similar velocities and diffusion coefficients which had a linear relationship with the incident laser fluence. In the other three environments tested (cell culture medium, serum and blood), this linear dependence could still be observed for both particles, but EM-JPMs under the same conditions moved significantly faster than the uncoated JPMs (Figure 4). Furthermore, relative velocities and relative diffusion coefficients were also calculated to compare the differences between bare JPMs and EM-JPMs, as shown in Figure S5. The maximum speed for the EM-JPMs reached $19.8 \mu \mathrm{m} / \mathrm{s}$ (PBS), $12.10 \mu \mathrm{m} / \mathrm{s}$ (medium), $3.52 \mu \mathrm{m} / \mathrm{s}$ (serum), and $2.33 \mu \mathrm{m} / \mathrm{s}$ (blood), which corresponds to relative speeds of nearly $3.96,2.42,0.7$, and 0.47 body lengths/s, respectively. Obviously, all particles move slower when in contact with a more viscous environment. However, after modification with the cell membrane, EM-JPMs moved faster and longer in the biological environments than the bare ones. In order to directly correlate this behavior to the antibiofouling effect, bare JPMs and membrane-modified JPMs were cultured 
in PBS solution, cell culture medium, serum, and blood at 37 ${ }^{\circ} \mathrm{C}$ for 1 and $6 \mathrm{~h}$. Then the morphology of the particles was characterized immediately with scanning electron microscopy (SEM). For better observation, templates were not removed. As shown in Figure S6, both the bare JPMs and EM-JPMs still maintained their Janus structure in PBS solution. However, the bare JPMs were aggregated together in cell culture medium, serum, and whole blood and showed a roughened surface (Figure S7). With the protection of the erythrocyte membrane, EM-JPMs were barely affected, which could be the reason that the movement of EM-JPMs in biological fluids was enhanced.

In order to be suitable for biomedical applications, the materials applied have to be biocompatible and biodegradable. Consequently, to ascertain the biocompatibility of EM-JPMs, a standard 3-(4,5-dimethylthiazol-2-yl)-2,5-diphenyltetrazolium bromide (MTT) assay was used to evaluate the proliferation of mice embryonic fibroblast cells (NIH/3T3), which were precultured with samples for $24 \mathrm{~h}$. As shown in Figure S8, EMJPMs exhibit excellent biocompatibility. Furthermore, the biodegradation of JPMs in the presence of PBS $(\mathrm{pH} 7.4)$ at $37{ }^{\circ} \mathrm{C}$ as a function of time $(1,3,6$, and $24 \mathrm{~h})$ was also evaluated. We performed SEM to observe the morphological changes for these specific time periods, as shown in Figure S9. Due to the usage of the natural polysaccharide ( $\mathrm{CHI}$ ) and glycosaminoglycan (Hep) as wall materials, JPMs exhibited good performance in biodegradability. Furthermore, weakened interactions between the LbL layers due to the charge shielding induced by PBS and the effect of osmotic pressure might result in the breakage of the capsules. ${ }^{46-48}$

To assess the efficacy of thrombolysis of EM-JPMs (Figure $5 \mathrm{~A}$ ), human fibrinogen was used as raw material to construct a thrombus model by application of thrombin. The process of fibrin polymerization was monitored by using a turbidity assay which measured the absorbance $(340 \mathrm{~nm})$ at $37{ }^{\circ} \mathrm{C}$ for $70 \mathrm{~min}$. The obtained column diagram, as shown in Figure 5B, reflected the assembly kinetics for thrombus formation, which reached its plateau around $60 \mathrm{~min}$. Consequently, the thrombus model used to assess thrombolysis was incubated for $60 \mathrm{~min}$ and characterized by SEM (Figure 5C). In order to visualize the efficacy of fibrinolysis, a fluorescent thrombus was generated by adding Alexa Fluor 488-conjugated human fibrinogen to the above-mentioned fibrinogen solution. The resulting fluorescent thrombus was analyzed immediately by CLSM. As shown in Figure 5D, a green fluorescent signal proved that a fluorescently labeled thrombus model was successfully established. To evaluate the efficacy of EM-JPMs for photothermal ablation of thrombus, the thrombus model was constructed by polymerizing fibrinogen on a $\mu$-Slide 8 well in the presence of $50 \mu \mathrm{L}$ of EM-JPMs $\left(\sim 4.9 \times 10^{5}\right.$ per $\left.\mu \mathrm{L}\right)$, followed by irradiation with a $760 \mathrm{~nm}$ NIR laser for $10 \mathrm{~min}\left(2.43 \mathrm{~J} / \mathrm{cm}^{2}\right)$. The whole process during thrombolytic therapy in situ was analyzed by CLSM. Time-lapsed CLSM images were recorded and are shown in Figure 5E. Here, the green fluorescence images represent the thrombus model (polymerized fibrinogen), and the red fluorescent signal originated from EM-JPMs. The results displayed destruction of the fibrin network with NIR laser irradiation as the fluorescence of thrombus was gradually released and spread to neighboring areas. The corresponding video can be found in the Supporting Information (Video S4), which shows the clot dissolution in real time. To demonstrate the combined function of motion and photothermal ablation, ${ }^{49,50}$ a fibrin clot was prepared by detaching the fibrin network and redispersing the thrombus in solution. Interestingly, by irradiation with the NIR laser, EMJPMs with red fluorescence were aggregated around the floating fibrin clot and then achieved efficient thrombolysis (Figure 5F and Video S5). In order to show that heparin was made available upon NIR-mediated heating, CLSM and SEM images of Janus capsules before and after NIR irradiation were taken (Figure S10). These images clearly displayed the disruption of the capsules, which in turn results in the release of heparin to enhance the photothermal-mediated thrombolysis. This is in line with previous publications in which it was demonstrated that laser-induced temperature increase could deconstruct the structural integrity of gold-incorporated polymers to achieve controlled drug release. ${ }^{51-54}$ To prove the anti-thrombus function of Hep, another control group was designed. As wall material, Hep was replaced with alginate to construct the EMJPMs, and these motors were also evaluated regarding their performance in photothermal ablation of thrombus at the same experimental conditions. As shown in Figure S11A and Video S6, thrombus models still maintained their initial state with a little fluorescence quenching after laser illumination. Furthermore, two other control groups were also investigated, including laser irradiation in the absence of EM-JPMs (Figure S11B and Video S7) and in the presence of (CHI/Hep) 5 capsules without gold coating (Figure S11C). Neither of them could induce the ablation of the thrombus. From these experiments, it was proven that only EM-JPMs prepared by Hep and CHI could achieve the efficient therapeutic effect toward thrombus ablation.

\section{CONCLUSION}

In summary, a photothermal thrombolytic platform based on erythrocyte membrane-coated Janus capsule micromotors was developed. Upon NIR irradiation, Janus capsules composed of biomolecules and partially covered with a gold shell were able to generate a local temperature gradient due to the strong surface plasmon resonance adsorption. The asymmetric structure of EM-JPMs induced rapid motion by thermophoretic force. The movement behavior of EM-JPMs could conveniently be modulated by the NIR laser power, as validated experimentally. By coating the JPMs with an erythrocyte membrane, the motors exhibited excellent performance in movement in relevant biological environments. In addition to providing a driving force, it was found that EM-JPMs could also successfully ablate thrombus by photothermal therapy upon NIR irradiation. This proof-of-concept study provides a motorbased therapeutic system with the advantages of active transportation, high efficacy, and biological compatibility.

\section{MATERIALS AND METHODS}

Materials. Poly(ethylene imine) solution $\left(M_{\mathrm{w}}=750000 \mathrm{~g} / \mathrm{mol}\right)$, chitosan (medium molecular weight), heparin sodium salt (from porcine intestinal mucosa), phenylmethanesulfonyl fluoride, sodium bicarbonate, ethylenediaminetetraacetic acid disodium salt dehydrate, rhodamine B isothiocyanate, sodium chloride, ammonium fluoride $\left(\mathrm{NH}_{4} \mathrm{~F}\right)$, and fibrinogen from human plasma were obtained from Sigma-Aldrich. Silica spheres with a diameter of $5 \mu \mathrm{m}$ were obtained from Microparticles GmbH, Germany. Protein BCA protein assay kit, Dulbecco's modified Eagle's medium (DMEM, high glucose), PBS (1 $\times, \mathrm{pH} 7.4$ ), cell membrane marker (Wheat germ agglutinin, Alexa Fluor 488 conjugate), thrombin, and Alexa Fluor 488-conjugated fibrinogen were purchased from ThermoFisher Scientific. MiniPROTEAN@TGX stain-free gels, $4 \times$ Laemmli protein sample buffer, and Precision Plus Protein All Blue Standards were obtained 
from Bio-Rad. Ultrapure water (Millipore) of $18.2 \mathrm{M} \Omega \cdot \mathrm{cm}$ was used for all experiments. All chemicals were used as received.

Preparation of Janus Polymeric Motors. CHI/Hep microcapsules were prepared by the LbL self-assembly method. ${ }^{55-57}$ Briefly, $1 \mathrm{mg} / \mathrm{mL}$ of $\mathrm{CHI}$ and Hep solution containing $0.1 \mathrm{M} \mathrm{NaCl}$ was alternatively deposited on the surface of template microparticles under continuous shaking for $15 \mathrm{~min}$. Unassembled polymer was removed by washing three times with $0.1 \mathrm{M} \mathrm{NaCl}$. Fluorescein-labeled $\mathrm{CHI} / \mathrm{Hep}$ microcapsules were synthesized by adding one layer of rhodamine $\mathrm{B}-$ $\mathrm{CHI}$ during the assembly process. ${ }^{58-60}$ In order to construct Janus capsule motors, a droplet of the $\mathrm{CHI} / \mathrm{Hep}$ microparticle solution was dropped on a hydrophilic silica slide to form a monolayer of microparticles. After evaporation in air, a turbo sputter coater (Quorum Technologies, K575X) was used to coat one side of the polymeric particles with a thin gold layer $(65 \mathrm{mV}, 30 \mathrm{~s})$. Ultrasound treatment was used to redisperse the Janus polymeric particles into aqueous solution. Next, $50 \mathrm{mg} / \mathrm{mL}$ of $\mathrm{NH}_{4} \mathrm{~F}$ solution was then used to dissolve the silica template to form Janus polymeric capsules with hollow structure. The morphology of JPMs was characterized with scanning electron microscopy (FEI Quanta 200, 3D FEG, ThermoFisher Scientific, USA) and confocal laser scanning microscopy (Zeiss, LSM510 META, Germany). EDX elemental mapping analysis of JPMs was obtained by SEM (Phenom ProX, The Netherlands).

Fabrication of Erythrocyte Membrane-Camouflaged JPMs. The erythrocyte membrane was isolated and prepared from peripheral blood of healthy mice (Balb/c, male) by following the published protocols. $^{61}$ The morphology of erythrocytes and the erythrocyte membrane was characterized by using SEM and CLSM. EM-JPMs were obtained according to a previously reported procedure. ${ }^{62}$ Briefly, JPMs were mixed with preobtained vesicle solution of erythrocyte membrane and then cocultured for $4 \mathrm{~h}$ under gentle shaking at $4{ }^{\circ} \mathrm{C}$. To remove excess vesicles, the resulting solution was washed by centrifugation three times. The as-prepared EM-JPMs were then characterized by DLS (Malvern Zetasizer Nano ZS), CLSM, BCA protein assay, and sodium dodecyl sulfate polyacrylamide gel electrophoresis (SDS-PAGE).

Biocompatibility and Biodegradability Assessment. Mouse embryonic fibroblast cells $(\mathrm{NIH} / 3 \mathrm{~T} 3)$ were selected for cytotoxicity tests. NIH/3T3 cells were cultured in DMEM medium supplemented with $10 \%$ fetal bovine serum (FBS) and $1 \%$ penicillin-streptomycin at $37{ }^{\circ} \mathrm{C}$ in an atmosphere of $5 \% \mathrm{CO}_{2}$ and $70 \%$ humidity. Exponentially growing cells were seeded onto a 96-well plate and incubated in cell culture medium until the cell concentration reached $\sim 90 \%$. After being washed with PBS, EM-JPMs with different volumes $(0-80 \mu \mathrm{L}, \sim 4.9 \times$ $10^{5}$ per $\mu \mathrm{L}$, counted by using a hemocytometer) were added, followed by the standard MTT assay. The biodegradability was tested by immersing JPMs into PBS solution (1×, $\mathrm{pH} 7.4)$ at $37^{\circ} \mathrm{C}$ for $1,3,6$, and $24 \mathrm{~h}$. The morphology was investigated by SEM immediately.

Motion Experiments and Analysis. For movement experiments, NIR laser propulsion was achieved by using a two-photon laser (Chameleon Vision, Coherent, USA) at $760 \mathrm{~nm}$ as the power source. The obtained JPMs and EM-JPMs were dispersed in PBS solution, cell culture medium, FBS, and blood. The motion frames of JPMs and EM-JPMs were recorded by CLSM (Zeiss, LSM510 META). All the motion experiments were performed at room temperature. The motion coordinate information, tracking trajectories, and further motion analysis were conducted by ImageJ. The mean square displacement was calculated according to the published method, MSD $=(x(\Delta t)-x(0))^{2}+(y(\Delta t)-y(0))^{2}{ }^{42-44}$ Diffusion coefficients $(D)$ were calculated according to the following equation, $D=\mathrm{MSD} /(i$ $\times \Delta t$ ), where $\Delta t$ is the time interval and $i$ is the dimensional index. ${ }^{42-44}$ Here, $i$ is equal to 4 for two-dimensional analysis.

Generation and Photothermal Ablation of Thrombus Models. Thrombus models were established by following the published protocols. ${ }^{63}$ Fibrinogen from human plasma $(1 \mathrm{mg} / \mathrm{mL})$ as the physiological source of thrombus was dissolved in a solution of $50 \mathrm{mM}$ Tris- $\mathrm{HCl}(\mathrm{pH} 7.4)$ and $140 \mathrm{mM} \mathrm{NaCl}$. Thrombus formation was induced by adding $1 \mathrm{U} / \mathrm{mL}$ thrombin and $2.5 \mathrm{mM} \mathrm{CaCl}_{2}$ to the fibrinogen solution, which was then cultured at $37{ }^{\circ} \mathrm{C}$ for $1 \mathrm{~h}$. A turbidity assay was used to monitor the process of thrombus formation by measuring the absorbance $(340 \mathrm{~nm})$ at $10 \mathrm{~min}$ intervals. Fluorescent-labeled thrombus was formed by an additional supply of $10 \mu \mathrm{L}$ of Alexa Fluor 488-conjugated fibrinogen to the fibrinogen solution containing thrombin and $\mathrm{CaCl}_{2}$, followed by incubation at 50 ${ }^{\circ} \mathrm{C}$ for $2 \mathrm{~h}$. The fluorescent thrombus was characterized by CLSM (Leica, TCS SP5X). Thrombus models and EM-JPMs $(50 \mu \mathrm{L}, \sim 4.9 \times$ $10^{5}$ per $\mu \mathrm{L}$ ) in a $\mu$-slide 8 well (ibidi, Germany) were irradiated with a $760 \mathrm{~nm}$ laser at a laser power of $2.43 \mathrm{~J} / \mathrm{cm}^{2}$ (two-photon laser, Chameleon Vision, Coherent, USA). Thrombus lysis was evaluated by a fluorescence-based assay.

\section{ASSOCIATED CONTENT}

\section{Supporting Information}

The Supporting Information is available free of charge on the ACS Publications website at DOI: 10.1021/acsnano.8b01772.

Additional images (PDF)

Video S1: Brownian motion of EM-JPMs without NIR illumination (AVI)

Video S2: Movement behavior of EM-JPMs upon NIR illumination (AVI)

Video S3: "Off/On” controllable motion of EM-JPMs (AVI)

Video S4: Photothermal ablation of thrombus in the presence of EM-JPMs which were prepared by $\mathrm{CHI}$ and Hep (AVI)

Video S5: Photothermal ablation of floating thrombus (AVI)

Video S6: Photothermal ablation of thrombus in the presence of EM-JPMs which were prepared by $\mathrm{CHI}$ and alginate (AVI)

Video S7: Thrombus models directly irradiated with NIR laser (AVI)

\section{AUTHOR INFORMATION}

\section{Corresponding Author}

*E-mail: J.C.M.v.Hest@tue.nl.

ORCID 1

David S. Williams: 0000-0002-8209-6899

Jan C. M. van Hest: 0000-0001-7973-2404

\section{Author Contributions}

J.S. and J.vH. conceived the project and designed the experiments. J.S. performed the experiments with the help of M.A., G.S., D.S.W., and S.C. J.S wrote the manuscript. J.vH. reviewed and edited the draft. All authors have given approval to the final version of the manuscript.

\section{Notes}

The authors declare no competing financial interest.

\section{ACKNOWLEDGMENTS}

The authors would like to acknowledge the ERC Advanced Grant Artisym 694120, the Dutch Ministry of Education, Culture and Science (Gravitation program 024.001.035), the China Scholarship Council and the European Union's Horizon 2020 research and innovation programme Marie SklodowskaCurie Innovative Training Networks (ITN) Nanomed, under Grant No. 676137, for funding.

\section{REFERENCES}

(1) Katuri, J.; Ma, X.; Stanton, M. M.; Sánchez, S. Designing Microand Nanoswimmers for Specific Applications. Acc. Chem. Res. 2017, 50, $2-11$. 
(2) Guix, M.; Mayorga-Martinez, C. C.; Merkoçi, A. Nano/ Micromotors in (Bio)chemical Science Applications. Chem. Rev. 2014, 114, 6285-6322.

(3) Wang, H.; Pumera, M. Fabrication of Micro/Nanoscale Motors. Chem. Rev. 2015, 115, 8704-8735.

(4) Wang, J.; Gao, W. Nano/Microscale Motors: Biomedical Opportunities and Challenges. ACS Nano 2012, 6, 5745-5751.

(5) Mhanna, R.; Qiu, F. M.; Zhang, L.; Ding, Y.; Sugihara, K.; Zenobi-Wong, M.; Nelson, B. J. Artificial Bacterial Flagella for Remote-Controlled Targeted Single-Cell Drug Delivery. Small 2014, 10, 1953-1957.

(6) Lin, X. K.; Wu, Z. G.; Wu, Y. J.; Xuan, M. J.; He, Q. SelfPropelled Micro-/Nanomotors Based on Controlled Assembled Architectures. Adv. Mater. 2016, 28, 1060-1072.

(7) Li, J. X.; de Ávila, B. E.; Gao, W.; Zhang, L. F.; Wang, J. Micro/ Nanorobots for Biomedicine: Delivery, Surgery, Sensing, and Detoxification. Sci. Robot. 2017, 2, eaam6431.

(8) Yánez-Sedeño, P.; Campuzano, S.; Pingarrón, J. M. Janus Particles for (Bio)Sensing. Appl. Mater. Today 2017, 9, 276-288.

(9) Wu, J.; Balasubramanian, S.; Kagan, D.; Manesh, K. M.; Campuzano, S.; Wang, J. Motion-Based DNA Detection Using Catalytic Nanomotors. Nat. Commun. 2010, 1, 36.

(10) Schroeder, A.; Heller, D. A.; Winslow, M. M.; Dahlman, J. E.; Pratt, G. W.; Langer, R.; Jacks, T.; Anderson, D. G. Treating Metastatic Cancer with Nanotechnology. Nat. Rev. Cancer 2012, 12, $39-50$.

(11) Hortelão, A. C.; Patiño, T.; Perez-Jiménez, A.; Blanco, À.; Sánchez, S. Enzyme-Powered Nanobots Enhance Anticancer Drug Delivery. Adv. Funct. Mater. 2017, 1705086.

(12) Xu, T. L.; Gao, W.; Xu, L. P.; Zhang, X. J.; Wang, S. T. FuelFree Synthetic Micro/Nanomachines. Adv. Mater. 2017, 29, 1603250.

(13) Xu, L. L.; Mou, F. Z.; Gong, H. T.; Luo, M.; Guan, J. G. LightDriven Micro/Nanomotors: From Fundamentals to Applications. Chem. Soc. Rev. 2017, 46, 6905-6926.

(14) Baraban, L.; Streubel, R.; Makarov, D.; Han, L. Y.; Karnaushenko, D.; Schmidt, O. G.; Cuniberti, G. Fuel-Free Locomotion of Janus Motors: Magnetically Induced Thermophoresis. ACS Nano 2013, 7, 1360-1367.

(15) Xu, T. L.; Xu, L. P.; Zhang, X. J. Ultrasound Propulsion of Micro-/Nanomotors. Appl. Mater. Today 2017, 9, 493-503.

(16) Mizrahy, S.; Peer, D. Polysaccharides as Building Blocks for Nanotherapeutics. Chem. Soc. Rev. 2012, 41, 2623-2640.

(17) Doshi, N.; Zahr, A. S.; Bhaskar, S.; Lahann, J.; Mitragotri, S. Red Blood Cell-Mimicking Synthetic Biomaterial Particles. Proc. Natl. Acad. Sci. U. S. A. 2009, 106, 21495-21499.

(18) Ren, X. Q.; Zheng, R.; Fang, X. L.; Wang, X. F.; Zhang, X. Y.; Yang, W. L.; Sha, X. Y. Red Blood Cell Membrane Camouflaged Magnetic Nanoclusters for Imaging-Guided Photothermal Therpay. Biomaterials 2016, 92, 13-24.

(19) Xuan, M. J.; Shao, J. X.; Dai, L. R.; He, Q.; Li, J. B. Macrophage Cell Membrane Camouflaged Mesoporous Silica Nanocapsules for In Vivo Cancer Therapy. Adv. Healthcare Mater. 2015, 4, 1645-1652.

(20) Luk, B. T.; Zhang, L. F. Cell Membrane-Camouflaged Nanoparticles for Drug Delivery. J. Controlled Release 2015, 220, 600-607.

(21) Xuan, M. J.; Shao, J. X.; Dai, L. R.; Li, J. B.; He, Q. Macrophage Cell Membrane Camouflaged Au Nanoshells for In Vivo Prolonged Circulation Life and Enhanced Cancer Photothermal Therapy. ACS Appl. Mater. Interfaces 2016, 8, 9610-9618.

(22) Dehaini, D.; Wei, X. L.; Fang, R. H.; Masson, S.; Angsantikul, P.; Luk, B. T.; Zhang, Y.; Ying, M.; Jiang, Y.; Kroll, A. V.; Gao, W. W.; Zhang, L. F. Erythrocyte-Platelet Hybrid Membrane Coating for Enhanced Nanoparticle Functionalization. Adv. Mater. 2017, 29, 1606209.

(23) Tan, S. W.; Wu, T. T.; Zhang, D.; Zhang, Z. P. Cell or Cell Membrane-Based Drug Delivery Systmes. Theranostics 2015, 5, 863881.
(24) Yoo, J. W.; Irvine, D. J.; Discher, D. E.; Mitragotri, S. BioInspired, Bioengineered and Biomimetic Drug Delivery Carriers. Nat. Rev. Drug Discovery 2011, 10, 521-535.

(25) Yurkin, S. T.; Wang, Z. J. Cell Membrane-Derived Nanoparticles: Emerging Clinical Opportunities for Targeted Drug Delivery. Nanomedicine 2017, 12, 2007-2019.

(26) Hu, C. M. J.; Fang, R. H.; Zhang, L. F. Erythrocyte-Inspired Delivery Systems. Adv. Healthcare Mater. 2012, 1, 537-547.

(27) Timin, A. S.; Litvak, M. M.; Gorin, D. A.; Atochina-Vasserman, E. N.; Atochin, D. N.; Sukhorukov, G. B. Cell-Based Drug Delivery and Use of Nano- and Microcarriers for Cell Functionalization. Adv. Healthcare Mater. 2018, 7, 1700818.

(28) Wu, Z. G.; Li, T. L.; Gao, W.; Xu, T. L.; Jurado-Sánchez, B.; Li, J. X.; Gao, W. W.; He, Q.; Zhang, L. F.; Wang, J. Cell-MembraneCoated Synthetic Nanomotors for Effective Biodetoxification. Adv. Funct. Mater. 2015, 25, 3881-3887.

(29) Wu, Z. G.; Li, J. X.; de Ávila, B. E.; Li, T. L.; Gao, W. W.; He, Q.; Zhang, L. F.; Wang, J. Water-Powered Cell-Mimicking Janus Micromotor. Adv. Funct. Mater. 2015, 25, 7497-7501.

(30) Li, J. X.; Angsantikul, P.; Liu, W. J.; de Ávila, B. E.; Chang, X. C.; Sandraz, E.; Liang, Y. Y.; Zhu, S. Y.; Zhang, Y.; Chen, C. R; Gao, W. W.; Zhang, L. F.; Wang, J. Biomimetic Platelet-Camouflaged Nanorobots for Binding and Isolation of Biological Threats. Adv. Mater. 2018, 30, 1704800 .

(31) Gao, W. W.; Zhang, L. F. Engineering Red-Blood-CellMembrane-Coated Nanoparticles for Broad Biomedical Applications. AIChE J. 2015, 61, 738-746.

(32) Hu, C. M. J.; Fang, R. H.; Luk, B. T.; Chen, K. N. H.; Carpenter, C.; Gao, W. W.; Zhang, K.; Zhang, L. F. 'Marker-of-Self' Functionalization of Nanoscale Particles through a Top-Down Cellular Membrane Coating Approach. Nanoscale 2013, 5, 2664-2668.

(33) Bunge, A.; Fischlechner, M.; Loew, M.; Arbuzova, A.; Herrmann, A.; Huster, D. Characterization of Lipid Bilayers Adsorbed on Spherical LbL-support. Soft Matter 2009, 5, 3331-3339.

(34) Krishna, G.; Shutava, T.; Lvov, Y. Lipid Modified Polyelectrolyte Microcapsules with Controlled Diffusion. Chem. Commun. 2005, 2796-2798.

(35) Xuan, M. J.; Mestre, R.; Gao, C. Y.; Zhou, C.; He, Q.; Sánchez, S. Noncontinuous Super-Diffusive Dynamics of Light-Activated Nanobottle Motor. Angew. Chem. Int. Ed. 2018, DOI: 10.1002/ anie.201801910.

(36) Skirtach, A. G.; Dejugnat, C.; Braun, D.; Susha, A. S.; Rogach, A. L.; Parak, W. J.; Möhwald, H.; Sukhorukov, G. B. The Role of Metal Nanoparticles in Remote Release of Encapsulated Materials. Nano Lett. 2005, 5, 1371-1377.

(37) Skirtach, A. G.; Muñoz Javier, A.; Kreft, O.; Köhler, K.; Piera Alberola, A.; Möhwald, H.; Parak, W. J.; Sukhorukov, G. B. LaserInduced Release of Encapsulated Materials inside Living Cells. Angew. Chem. Int. Ed. 2006, 45, 4612-4617.

(38) Delcea, M.; Sternberg, N.; Yashchenok, A. M.; Georgieva, R.; Bäumler, H.; Möhwald, H.; Skirtach, A. G. Nanoplasmonics for DualMolecule Release through Nanopores in the Membrane of Red Blood Cells. ACS Nano 2012, 6, 4169-4180.

(39) Skirtach, A. G.; Karageorgiev, P.; De Geest, B. G.; Pazos-Perez, N.; Braun, D.; Sukhorukov, G. B. Nanorods as Wavelength-Selective Absorption Centers in the Visible and Near-Infrared Regions of the Electromagnetic Spectrum. Adv. Mater. 2008, 20, 506-510.

(40) Lin, X. K.; Si, T. Y.; Wu, Z. G.; He, Q. Self-Thermophoretic Motion of Controlled Assembled Micro-/Nanomotors. Phys. Chem. Chem. Phys. 2017, 19, 23606-23613.

(41) Weissleder, R. A Clearer Vision for In Vivo Imaging. Nat. Biotechnol. 2001, 19, 316-317.

(42) Xuan, M. J.; Wu, Z. G.; Shao, J. X.; Dai, L. R.; Si, T. Y.; He, Q. Near Infrared Light-Powered Janus Mesoporous Silica Nanoparticle Motors. J. Am. Chem. Soc. 2016, 138, 6492-6497.

(43) Ma, X.; Hahn, K.; Sanchez, S. Catalytic Mesoporous Janus Nanomotors for Active Cargo Delivery. J. Am. Chem. Soc. 2015, 137, 4976-4979. 
(44) Abdelmohsen, L. K. E. A.; Nijemeisland, M.; Pawar, G. M.; Janssen, G. A.; Nolte, R. J. M.; van Hest, J. C. M.; Wilson, D. A. Dynamic Loading and Unloading of Proteins in Polymeric Stomatocytes: Formation of an Enzyme-Loaded Supramolecular Nanomotor. ACS Nano 2016, 10, 2652-2660.

(45) Rao, L.; Meng, Q. F.; Bu, L. L.; Cai, B.; Huang, Q. Q.; Sun, Z. J.; Zhang, W. F.; Li, A.; Guo, S. S.; Liu, W.; Wang, T. H.; Zhao, X. Z. Erythrocyte Membrane-Coated Upconversion Nanoparticles with Minimal Protein Adsorption for Enhanced Tumor Imaging. ACS Appl. Mater. Interfaces 2017, 9, 2159-2168.

(46) De Geest, B. G.; Sanders, N. N.; Sukhorukov, G. B.; Demeester, J.; De Smedt, S. C. Release Mechanisms for Polyelectrolyte Capsules. Chem. Soc. Rev. 2007, 36, 636-649.

(47) Angelova, N.; Hunkeler, D. Permeability and Stability of Chitosan-Based Capsules: Effect of Preparation. Int. J. Pharm. 2002, 242, 229-232.

(48) Peyratout, C. S.; Dähne, L. Tailor-Made Polyelectrolyte Microcapsules: From Multilayers to Smart Containers. Angew. Chem. Int. Ed. 2004, 43, 3762-3783.

(49) Xuan, M. J.; Shao, J. X.; Lin, X. K.; Dai, L. R.; He, Q. SelfPropelled Janus Mesoporous Silica Nanomotors with Sub-100 nm Diameters for Drug Encapsulation and Delivery. ChemPhysChem 2014, $15,2255-2260$.

(50) Gao, W. W.; de Ávila, B. E.; Zhang, L. F.; Wang, J. Targeting and Isolation of Cancer Cells Using Micro/Nanomotors. Adv. Drug Delivery Rev. 2017, DOI: 10.1016/j.addr.2017.09.002.

(51) Carregal-Romero, S.; Ochs, M.; Rivera-Gil, P.; Ganas, C.; Pavlov, A. M.; Sukhorukov, G. B.; Parak, W. J. NIR-Light Triggered Delivery of Macromolecules into the cytosol. J. Controlled Release 2012, 159, 120-127.

(52) Ochs, M.; Carregal-Romero, S.; Rejman, J.; Braeckmans, K.; De Smedt, S. C.; Parak, W. J. Light-Addressable Capsules as Caged Compound Matrix for Controlled Triggering of Cytosolic Reactions. Angew. Chem. Int. Ed. 2013, 52, 695-699.

(53) Muñoz Javier, A.; del Pino, P.; Bedard, M. F.; Ho, D.; Skirtach, A. G.; Sukhorukov, G. B.; Plank, C.; Parak, W. J. Photoactivated Release of Cargo from the Cavity of Polyelectrolyte Capsules to the Cytosol of Cells. Langmuir 2008, 24, 12517-12520.

(54) Hühn, D.; Govorov, A.; Gil, P. R.; Parak, W. J. Photostimulated $\mathrm{Au}$ Nanoheaters in Polymer and Biological Media: Characterization of Mechanical Destruction and Boiling. Adv. Funct. Mater. 2012, 22, 294-303.

(55) Decher, G. Fuzzy Nanoassemblies: Toward Layered Polymeric Multicomposites. Science 1997, 277, 1232-1237.

(56) Donath, E.; Sukhorukov, G. B.; Caruso, F.; Davis, S. A.; Möhwald, H. Novel Hollow Polymer Shells by Colloid-Templated Assembly of Polyelectrolytes. Angew. Chem. Int. Ed. 1998, 37, 22012205.

(57) Caruso, F.; Caruso, R. A.; Möhwald, H. Nanoengineering of Inorganic and Hybrid Hollow Spheres by Colloidal Templating. Science 1998, 282, 1111-1114.

(58) Richert, L.; Lavalle, P.; Payan, E.; Shu, X. Z.; Prestwich, G. D.; Stoltz, J.; Schaaf, P.; Voegel, J.; Picart, C. Layer by Layer Buildup of Polysaccharide Films: Physical Chemistry and Cellular Adhesion Aspects. Langmuir 2004, 20, 448-458.

(59) Ma, O.; Lavertu, M.; Sun, J.; Nguyen, S.; Buschmann, M. D.; Winnik, F. M.; Hoemann, C. D. Precise Derivatization of Structurally Distinct Chitosans with Rhodamine B Isothiocyanate. Carbohydr. Polym. 2008, 72, 616-624.

(60) Ren, Y.; Xie, H. G.; Liu, X. C.; Yang, F.; Yu, W. T.; Ma, X. J. Tuning the Formation and Stability of Microcapsules by Environmental Conditions and Chitosan Structure. Int. J. Biol. Macromol. 2016, 91, 1090-1100.

(61) Gao, W. W.; Hu, C. M. J.; Fang, R. H.; Luk, B. T.; Su, J.; Zhang, L. F. Surface Functionalization of Gold Nanoparticles with Red Blood Cell Membranes. Adv. Mater. 2013, 25, 3549-3553.

(62) Hu, C. M. J.; Zhang, L.; Aryal, S.; Cheung, C.; Fang, R. H.; Zhang, L. F. Erythrocyte Membrane-Camouflaged Polymeric Nano- particles as a Biomimetic Delivery Platform. Proc. Natl. Acad. Sci. U. S. A. 2011, 108, 10980-10985.

(63) Singh, N.; Varma, A.; Verma, A.; Maurya, B. N.; Dash, D. Relief from Vascular Occlusion using Photothermal Ablation of Thrombus with a Multimodal Perspective. Nano Res. 2016, 9, 2327-2337. 